\title{
Higgs Boson Sensitivity in ATLAS
}

\author{
Jana Novakova*† \\ Charles University in Prague, Faculty of Mathematics and Physics, Institute of Particle and \\ Nuclear Physics \\ E-mail: jana.novakova@cern.ch
}

Higgs boson searches are one of the main goals of the ATLAS physics program. Higgs boson exclusion limits, expected with an integrated luminosity of $1 \mathrm{fb}^{-1}$ in proton-proton collisions at the centre of mass energy of $7 \mathrm{TeV}$, are shown. This integrated luminosity can be reached by the end of year 2011 according to the LHC plans. The expected sensitivity for both, the Standard Model Higgs boson and the MSSM Higgs bosons, is discussed. The results are based on the full Monte Carlo simulations rescaled from 10 and $14 \mathrm{TeV}$ studies [1, 2].

Workshop on Discovery Physics at the LHC-Kruger 2010

December 05-10, 2010

Kruger National Park, Mpumalanga, South Africa

\footnotetext{
*Speaker.

$\dagger$ On the behalf of the ATLAS Collaboration
} 


\section{Introduction}

One of the open points in particle physics is the mechanism how particles acquire their masses. This might be explained by electroweak symmetry breaking via Higgs mechanism which leads to the existence of a scalar boson in the Standard Model (SM). The same mechanism might be also applied in models beyond the Standard Model, e.g Minimal Supersymmetric Model where it results in five physical states (three neutral $h / H / A$ and two charged $H^{+} / H^{-}$Higgs bosons).

Despite the Higgs boson searches at LEP and Tevatron colliders, no Higgs boson particle have been observed so far. The searches have led to limits on the Higgs boson mass: LEP excluded the SM Higgs particle below $M_{H}<114.4 \mathrm{GeV}$ [3], latest Tevatron results have led to the exclusion in the mass range $158-175 \mathrm{GeV}$ and $100-109 \mathrm{GeV}$ at $95 \%$ C.L. [4]. The Higgs boson searches have started recently also in the ATLAS experiment at the LHC. The prospects of the sensitivity for integrated luminosity of $1 \mathrm{fb}^{-1}$ in proton-proton collisions at the center of mass energy of $7 \mathrm{TeV}$ are discussed in this paper.

\section{Standard Model Higgs boson}

Details about three important SM Higgs boson channels and the expected exclusion limits for the combination of several decay modes are shown in this section.

\section{1 $H \rightarrow W W^{(*)}$}

The Higgs boson decay into a pair of $W$ bosons gives the highest sensitivity for the SM Higgs boson in the range between 120 and $200 \mathrm{GeV}$ [2]. It profits from a high $H \rightarrow W W^{(*)}$ branching ratio and a very good electron and muon identification in the ATLAS experiment. The searches are performed separately for events with 0,1 or 2 accompanying jets to maximize the sensitivity. The dominant background processes are the $W W^{(*)}$ and $W+$ jets (where a jet is mis-identified as a lepton) in the 0 jets analysis, $W W+$ jets and $t \bar{t}$ in the 1 jet analysis and $t \bar{t}$ in the 2 jets analysis.
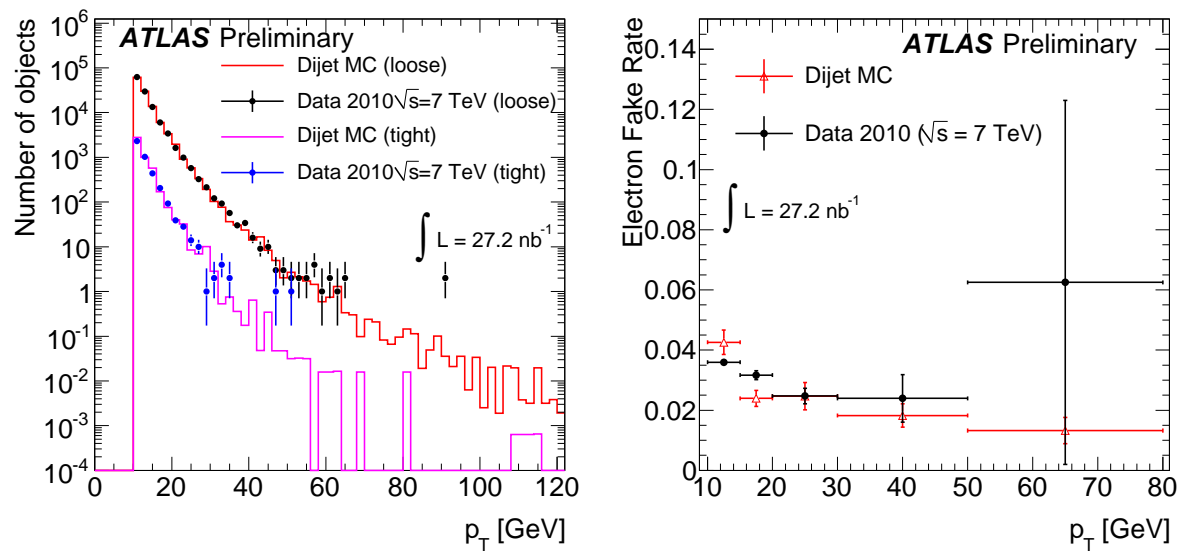

Figure 1: Transverse momentum spectrum of the "loose" and "tight" electrons in the dijet events on the left hand side and electron fake rates on the right hand side. 
The measurement of the $W+$ jets background was performed with $311 \mathrm{nb}^{-1}$ of data collected by ATLAS [5]. Any lepton not originating from the $W$ or $Z$ boson decay is considered as a fake lepton in the analysis. The fake lepton rate is defined as $f_{\ell} \equiv N_{\text {tight }} / N_{\text {loose }}$ in the Loose Lepton Method [6]. The "loose" lepton candidates are much less stringently defined than the "tight" candidates and are dominated by jets in most processes at the LHC. In other words, this fake rate is the probability that a loose object, that is likely to fake a lepton, also passes the full selection to be classified as a "tight" object. The fake rate is measured in the dijet events where a veto against $W / Z$ events is applied. A good agreement between data and Monte Carlo predictions is observed, see Figure 1 where the electron fake rate is shown.

The $H \rightarrow W W^{(*)}$ channel alone can exclude the Higgs boson at the $95 \%$ confidence level in a large mass range with an integrated luminosity of $1 \mathrm{fb}^{-1}$ as can be seen in Figure 2 .

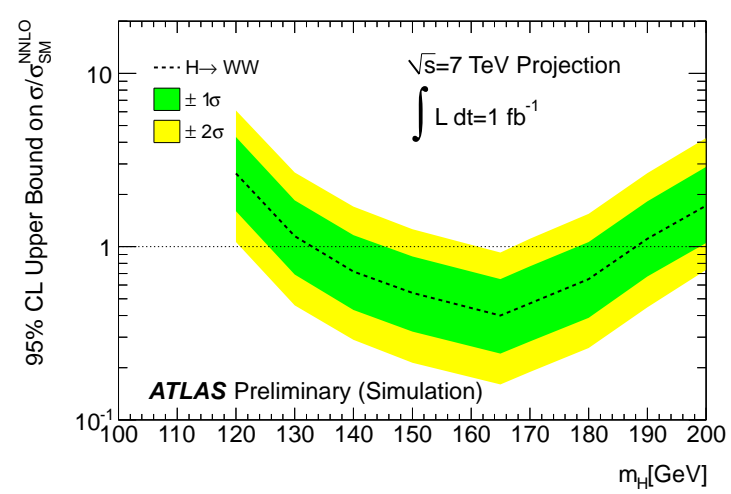

Figure 2: Expected $H \rightarrow W W^{(*)}$ exclusion limits with $1 \mathrm{fb}^{-1}$ of integrated luminosity.

\section{$2.2 H \rightarrow Z Z^{(*)}$}

The channel with both $Z$ bosons decaying into a pair of leptons provides the cleanest signature of all channels. A very good energy and momentum resolution for electrons and muons leads to a narrow Higgs boson mass peak. The main background comes from the irreducible $Z Z^{(*)} \rightarrow 4$ leptons decay. The reducible background $(Z+j e t s$ and $t \bar{t})$ is strongly suppressed if both $\mathrm{Z}$ bosons are on-shell (for the Higgs boson mass larger than $180 \mathrm{GeV}$ ). The reducible background plays an important role below $180 \mathrm{GeV}$. Strict calorimeter and track isolation requirements are applied in order to suppress this background. The analysis is most sensitive for a SM Higgs boson mass around $200 \mathrm{GeV}$ where un upper bound in the cross section times branching ratio of 1.3 times the SM cross section is expected with $1 \mathrm{fb}^{-1}$ of integrated luminosity [1].

If the Higgs boson mass is larger than $200 \mathrm{GeV}$, two other $Z Z$ decay modes contribute significantly in the sensitivity prospects: $H \rightarrow Z Z \rightarrow \ell^{+} \ell^{-} v_{\ell} \bar{v}_{\ell}$ and $H \rightarrow Z Z \rightarrow \ell^{+} \ell^{-} b \bar{b}$. The first channel alone leads to a small exclusion region $M_{H} \sim 400 \mathrm{GeV}$ with $1 \mathrm{fb}^{-1}$ of integrated luminosity [1].

\section{$2.3 H \rightarrow \gamma \gamma$}

The decay of the Higgs boson into two photons is an important channel in the Higgs boson 
low mass region, but has a very low branching ratio $\left(B R(H \rightarrow \gamma \gamma) \approx 0.002\right.$ for $110 \mathrm{GeV}<\mathrm{M}_{\mathrm{H}}<$ $140 \mathrm{GeV}$ ) [2]. Moreover, the background level is very high. The major background processes are irreducible background from the Born process $q \bar{q} \rightarrow \gamma \gamma$, the bremsstrahlung process $q g \rightarrow q \gamma \gamma$ and the box process $g g \rightarrow \gamma \gamma$. The reducible background arises from the inclusive prompt-photon $(\gamma+$ jet $)$ and multi-jet processes.

The expected limits were obtained by fitting the side-bands of the diphoton invariant mass $\left(M_{\gamma \gamma}\right)$ and are competitive with current CDF and D0 results [7,8]. The $M_{\gamma \gamma}$ was the only discriminating parameter used in this analysis for the sake of simplicity and robustness. Better sensitivity is expected with more advanced analysis (e.g using more discriminating variables and separating the events into categories depending on the numbers of jets, photon acceptance regions with different energy resolution and photon conversions).

\subsection{Expected exclusion limits}

The results from all aforementioned channels and including $H \rightarrow \tau^{+} \tau^{-}$and $H \rightarrow b \bar{b}$ decay modes are combined [1] using the profile likelihood ratio method [9], see Figure 3. The expected exclusion mass region at $95 \%$ confidence level with an integrated luminosity of $1 \mathrm{fb}^{-1}$ at the centre of mass energy of $7 \mathrm{TeV}$ is between 129 and $460 \mathrm{GeV}$. If the centre of mass energy is raised to $8 \mathrm{TeV}$ and the same integrated luminosity is considered, the upper limit improves significantly (525 GeV) compared to $7 \mathrm{TeV}$. Further improvements in the ATLAS analysis are foreseen, e.g moving to multivariate techniques for the particle identification and for the event selection. The expected exclusion regions are significantly larger than current Tevatron limits [4].
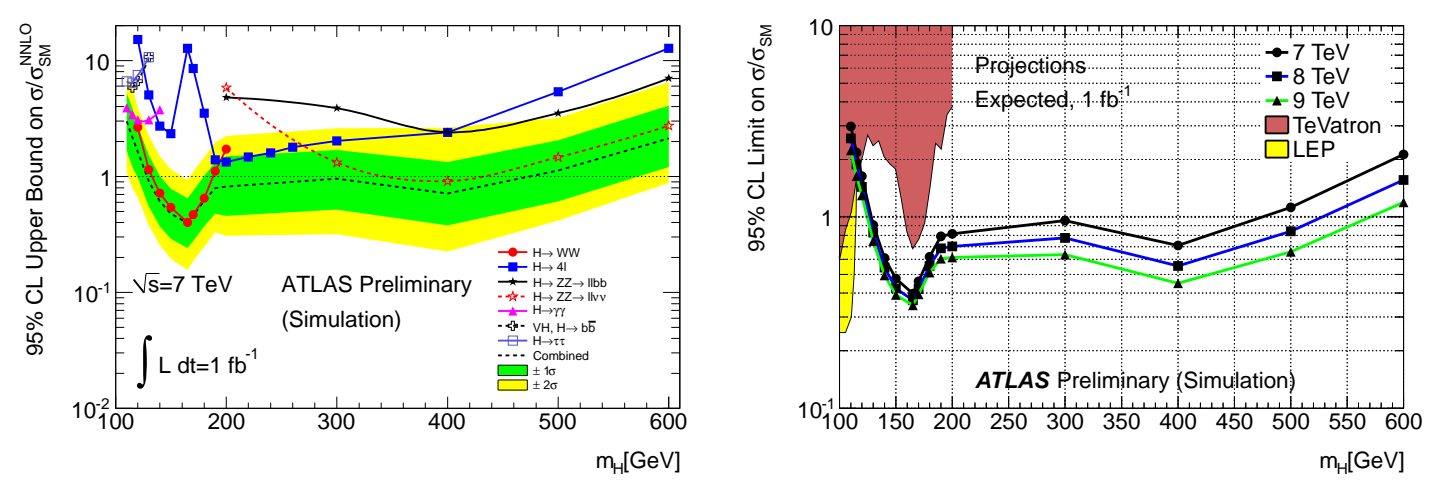

Figure 3: Expected exclusion limits with $1 \mathrm{fb}^{-1}$ of integrated luminosity: Contribution of different channels on the left hand side, exclusion limits for various center of mass energies on the right hand side.

\section{Minimal Supersymmetric Standard Model (MSSM) Higgs bosons}

Prospects of the charged Higgs boson searches and the neutral Higgs boson decay mode $H / A \rightarrow \mu^{+} \mu^{-}$in the MSSM model are briefly discussed in this section. 


\subsection{Charged Higgs boson}

If the charged Higgs boson mass is lower than the top mass, the Higgs boson can be produced in the decay of the on-shell top quark. This case is considered in the analysis.

The decay mode $H^{+} \rightarrow c \bar{s}$ contributes especially for $\tan \beta<1$ where it may reach $40 \%$ in the MSSM. The search for this channel is performed in the semi-leptonic $t \bar{t}$ events. The largest background contribution comes from $W$ bosons in the $t \bar{t}$ events, therefore a good separation between $W$ and $H^{+}$mass peaks plays an important role. The dijet mass resolution is improved by reconstructing the entire $t \bar{t}$ event using a kinematic fitter [10].

$H^{+} \rightarrow \tau^{+} v_{\tau}$ is the dominant decay mode for $\tan \beta>1$. It is searched for in the dilepton $t \bar{t}$ events. The helicity angle $\cos \theta_{l}^{*}$ and the generalized transverse mass $m_{\mathrm{T}_{2}}^{H^{+}}$were found to be a powerfull discriminant variables [10]. The dominant background also comes from $t \bar{t}$ events.

The expected upper limits on $B R\left(t \rightarrow b H^{+}\right)$with $1 \mathrm{fb}^{-1}$ of integrated luminosity at $95 \%$ C.L. [2] are shown in Figure 4 for two cases (assumming $B R\left(H^{+} \rightarrow c \bar{s}\right)=1$ and $B R\left(H^{+} \rightarrow \tau^{+} v_{\tau}\right)=$ $1)$.
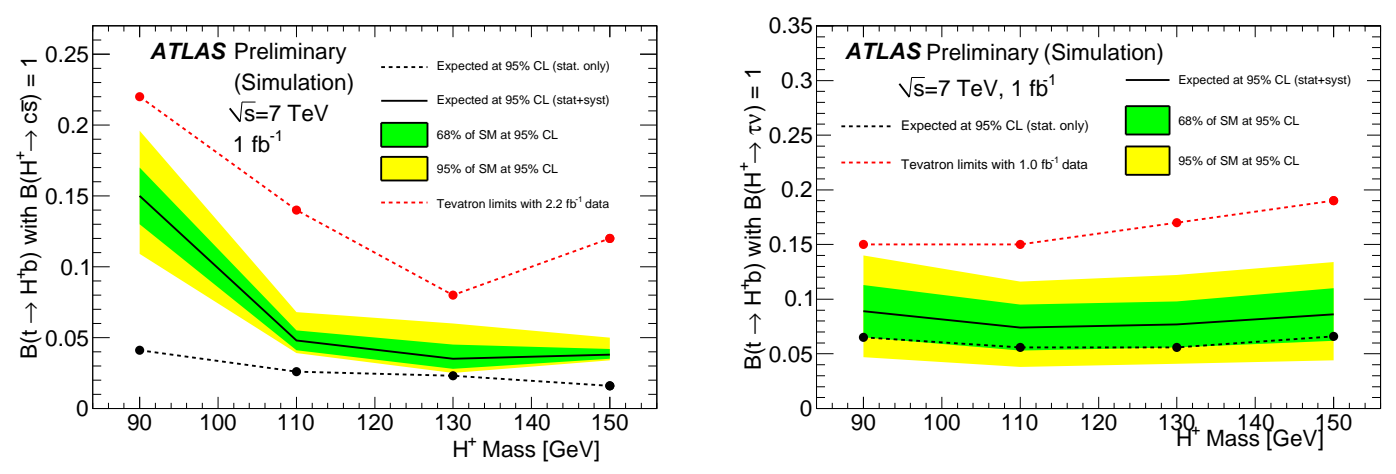

Figure 4: Expected MSSM charged Higgs upper limits on $B R\left(t \rightarrow b H^{+}\right)$with $1 \mathrm{fb}^{-1}$ of integrated luminosity assuming $B R\left(H^{+} \rightarrow c \bar{s}\right)=1$ on the left hand side and assuming $B R\left(H^{+} \rightarrow \tau^{+} v_{\tau}\right)=1$ on the right hand side.

\subsection{Neutral Higgs boson: $H / A \rightarrow \mu^{+} \mu^{-}$}

The channel $H / A \rightarrow \mu^{+} \mu^{-}$plays an important role in the high $\tan \beta$ region where the associated production of $H / A$ with $b$ quarks is dominant.

The dominant background process is the Drell-Yan $Z\left(\rightarrow \mu^{+} \mu^{-}\right)$production which is strongly suppressed by requiring the presence of at least one $b$-jet in the final state. However, the contribution from the $Z$ production in association with light jets is still present due to the mis-identification of light jets as a $b$-jets. In addition, there is the irreducible $Z$ production in association with the $b$-jets. Background from $t \bar{t}$ events also contributes significantly, but it can be reduced by requiring a low missing transverse energy and a low jet activity in the event.

Exclusion at $95 \%$ C.L. of $\tan \beta$ above 50 for the $M_{A}$ between 130 and $150 \mathrm{GeV}$ with integrated luminosity of $1 \mathrm{fb}^{-1}$ is expected [2] as shown in Figure 5. 

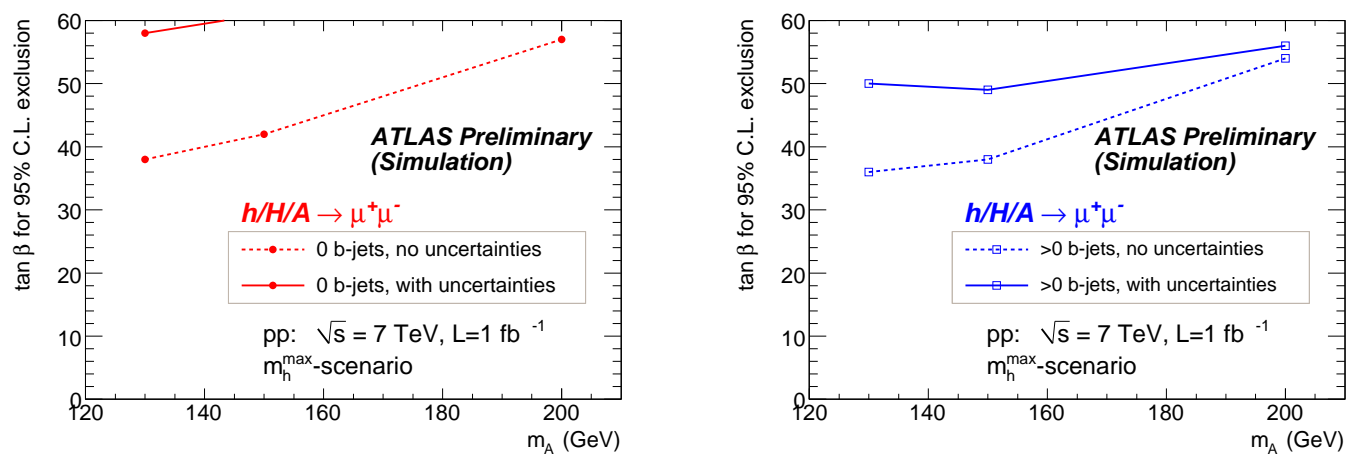

Figure 5: Expected MSSM $H / A \rightarrow \mu \mu$ exclusion limits with $1 \mathrm{fb}^{-1}$ of integrated luminosity.

\section{Acknowledgments}

The author is indebted to numerous ATLAS colleagues for their kind help and discussion while preparing this contribution, especially to Tomas Davidek, Bruce Mellado Garcia, William Murray and Chris Potter. This work was supported in part by grants MSM0021620859 and LA08032 of Ministry of Education, Youth and Sports of the Czech Republic.

\section{References}

[1] The Atlas Collaboration, ATLAS Sensitivity Prospects for 1 Higgs Boson Production at the LHC Running at 7, 8 or 9 TeV, ATL-PHYS-PUB-2010-015.

[2] The Atlas Collaboration, ATLAS Sensitivity Prospects for Higgs Boson Production at the LHC Running at $7 \mathrm{TeV}$, ATL-PHYS-PUB-2010-009.

[3] ALEPH, DELPHI, L3 and OPAL Collaborations, The LEP Electroweak Working Group, http://lepewwg.web.cern.ch/LEPEWWG/.

[4] The CDF Collaboration, The D0 Collaboration, the Tevatron New Physics, Higgs Working Group, Combined CDF and DO Upper Limits on Standard Model Higgs-Boson Production with up to 6.7 $\mathrm{fb}^{-1}$ of Data, FERMILAB-CONF-10-257-E, arXiv:1007.4587v1 [hep-ex].

[5] The ATLAS Collaboration Observation of the Background from $W+j e t s$ to the $H \rightarrow W W \rightarrow l l v v$ Search with the ATLAS detector at 7 TeV, ATL-CONF-2010-092.

[6] The ATLAS Collaboration Prospects for Higgs Boson Searches using the $H \rightarrow W W^{(*)} \rightarrow \ell v \ell v$ Decay Mode with the ATLAS Detector for $10 \mathrm{TeV}$, ATL-PHYS-PUB-2010-005.

[7] CDF Collaboration, Search for a SM Higgs Boson with the Diphoton Final State at CDF, CDF/PUB/EXOTIC/PUBLIC/10065.

[8] D0 Collaboration, Search for the SM Higgs Boson in gamma-gamma final states at DO with $L=4.2 \mathrm{fb}^{-1}$ data, D0 Note 5858-Conf.

[9] The ATLAS Collaboration, Expected Performance of the ATLAS Experiment Detector, Trigger and Physics, CERN-OPEN-2008-020, arXiv:0901.0512.

[10] The Atlas Collaboration, Expected Sensitivity in Light Charged Higgs Boson Searches for $H^{+}$to $\tau+v$ and $H^{+}$to $c+\bar{s}$ with Early LHC Data at the ATLAS Experiment, ATL-PHYS-PUB-2010-006. 